\title{
A Statistical Significance Test of the Difference in b-walue between Two Earthquake Groups
}

\author{
BY \\ Tokuji UTSU \\ Department of Geophysics, Faculty of Science \\ Hoklkaido University, Sapporo, Japan
}

\begin{abstract}
The probability density function for the value of $b$ in the formula $\log n(M)=a-b M$ estimated by the author's method has been obtained in an exact form. This function leads to a simple method to test the statistical significance of the difference between two $b$-values. Application of this method to SuYbHizo's data on foreshocks and aftershocks of the great Chilean earthquake of 1960 indicates a significant difference in $b$-value between the foreshocks and the aftershocks at the $98 \%$ confidence level.
\end{abstract}

Some seismologists (e.g., Mryamura, 1962a; SUYEHIRO, 1966) suggested the variation with space or time in the coefficient $b$ of the GUTENBERG-RICHTER's empirical formula

$$
\log n(M)=a-b M
$$

expressing the magnitude-frequency relationship of earthquake occurrence. However, since the value of $b$ cletermined from observational clata are subject to errors of various kinds, special care must be taken in comparing the $b$-values between different earthquake groups. Although some possible sources of errors have been discussed elsewhere (UTSU, 1967), it is assumed in this paper that every earthquake can be regarderl as a random sample from a population whose magnitude distribution takes the form as given by Equation (1). In this case the distribution of the estimated $b$-value around the value in population is controlled by the laws of probabilities, and it may be possible to obtain the functional forms of the distribution for some of the methods of estimation.

UTSU (1965, 1967) proposed a method for estimating the $b$-value from a given set of magnitude-frequency data, which used the formula

$$
b=\frac{s \log e}{\sum M_{i}-s M_{s}}
$$

where $s$ is the total number of earthquakes with magnitude greater than or equal to $M_{\text {s }}$ and $\sum M_{i}$ is the sum of the magnitudes of all the $s$ earthquakes. If the magnitucle is given at intervals of $\Delta M$, say 0.1 or $1 / 4$, and the central value for the smallest class is $\bar{M}_{s}, M_{s}$ should be taken as equal to $\bar{M}_{\mathrm{s}}-\Delta M / 2$. The accuracy of this method was compared with several conventional methods (e.g., the method of least squares) by using the Monte Carlo method, and it was found that the Equation (2) gives the most accurate and unbiased $b$-value when $b \Delta M$ is smaller than about $1 / 4$. When $b \Delta M$ is larger than about $1 / 4$, Equation (2) gives a systematically smaller value, but reduction to the correct value can be made by multiplying $\eta$ which is a function of $b \Delta M$ as shown in Table I (for details, see UTSU, 1967).

Table I. $\eta$ as a function of $b J M$.

\begin{tabular}{c|c|c|c}
\hline$\Delta M M$ & $\eta$ & $b \Delta M$ & $\gamma$ \\
\hline 0.1 & 1.001 & 0.6 & 1.154 \\
0.2 & 1.017 & 0.7 & 1.203 \\
0.9 & 1.039 & 0.8 & 1.268 \\
0.4 & 1.070 & 0.9 & 1.344 \\
0.5 & 1.108 & 1.0 & 1.407 \\
\hline
\end{tabular}

AkI (1965) noted that the $l$-value calculated by this formula was nothing but the maximum. likelihood estimate and tabulated its confidence limits for large values of s using the central limit theorem. In this paper the exact 
probability density function for the $b$-value calculated by this formula is obtained for any number of $s$, and a method is described to test the statistical significance of the difference in the $b$-value between two earthquake groups at a given confidence level.

If we deal with earthquakes with magnitude $M_{s}$ and larger, and convert magnitude $M$ to $M^{\prime}$ by putting

$$
M^{\prime}=M-M_{s},
$$

the probability that the $M^{\prime}$-value for an earthquake falls in the range from $X$ to $(X+d X)$ is given by

$\left.p(X) d X=b_{0} \ln 10 \exp \left(-b_{0} \ln 10 X\right) d X, \quad X \geq 0\right\}$ $p(X) d X=0$

where $b_{0}$ is the $b$-value in population. The moment generating function (m.g.f.) for this distribution takes the form

$$
q(z)=\int_{0}^{\infty} e^{z x} p(X) d X=\frac{1}{1-\frac{z}{b_{0} \ln 10}} .
$$

If $P(Y) d Y$ denotes the probability that $\sum M_{i}{ }^{\prime}\left(=\sum M_{i}-s M_{s}\right)$ takes a value between $Y$ and $Y+d Y$, its m.g.f. becomes

$$
Q(z)=q^{8}(z)=\frac{1}{\left(1-\frac{z}{b_{0} \ln 10}\right)^{8}}
$$

which is apparently the m.g.f. for a gamma distribution in the form

$$
P(Y) d Y=\frac{\left(b_{0} \ln 10\right)^{s}}{\Gamma(s)} \exp \left(-b_{0} \ln 10 Y\right) Y^{s-1} d Y .
$$

Therefore the distribution of $b$-value calculated by Equation (2) (i.e., $\left.b=s / \sum M_{i}^{\prime} \ln 10\right)$ ) is given by

$$
f(b) d b=\frac{s^{s}}{\Gamma(s)}\left(\frac{b_{0}}{b}\right)^{s+1} \exp \left(-\frac{s b_{0}}{b}\right) \frac{d b}{b_{0}} .
$$

Figures 1 and 2 represent the cumulative probability distributions of $b_{0} / b$ and $b / b_{0}$ (in $\%$ ) calculated by this equation for various values of $s$ from 7 to 3000 . Curves for $s>50$ in Figure 1 are in good agreement with Akr's tabulation.

Putting

$$
\chi^{2}=2 s b_{0} / b \text {, and } \phi=2 s,
$$

we obtain for the distribution of $\chi^{2}$,

$$
g\left(\chi^{2}\right) d \chi^{2}=\frac{1}{2^{\phi / 2}(\phi / 2)}\left(\chi^{2}\right)^{\phi / 2-1} \exp \left(-\chi^{2} / 2\right) d \chi^{2} .
$$

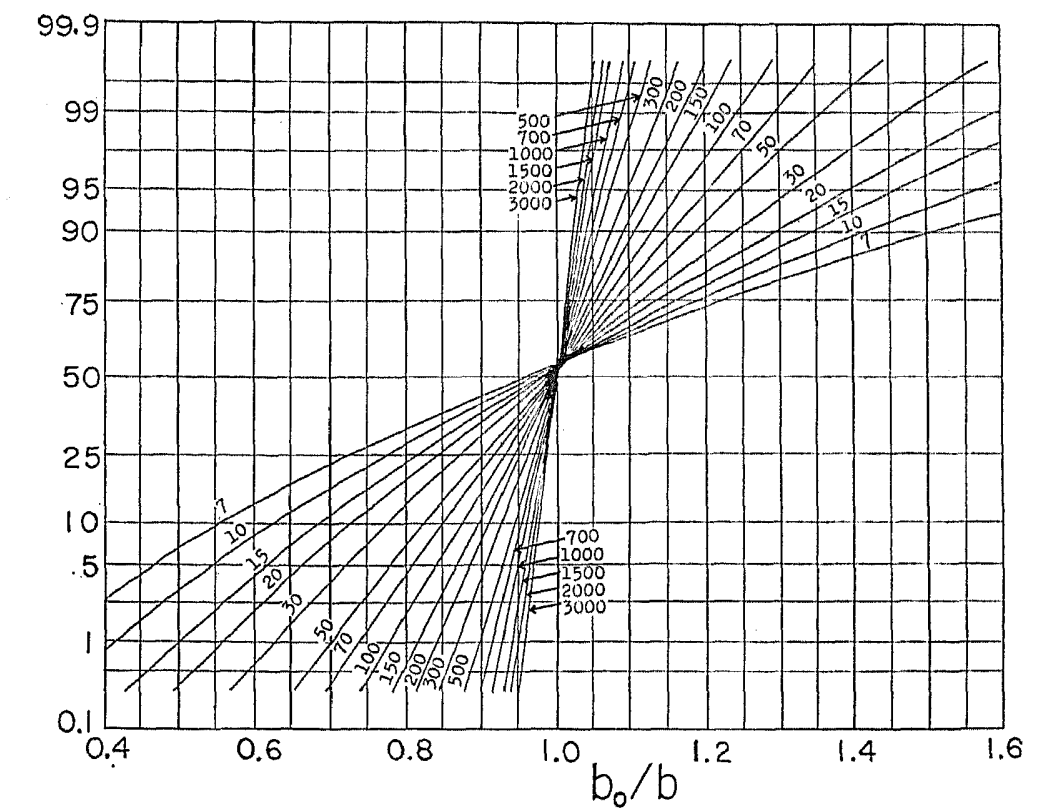

Fig. 1. Cumulative distribution of $b_{0} / b$ plotted on probability paper for $s=7,10, \cdots, 3000$. 


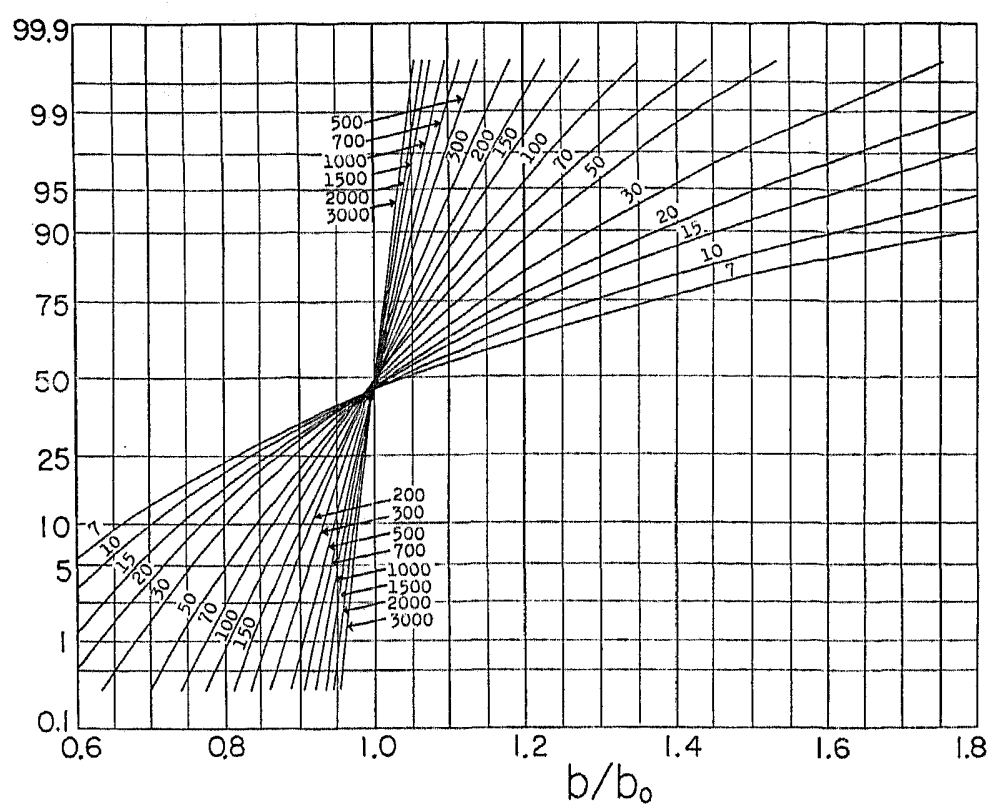

Fig. 2. Cumulative distribution of $b / b_{0}$ plotted on probability paper for $s=7,10, \cdots, 3000$.

This is the well-known $\chi^{2}$-distribution with $\phi$ degrees of freedom.

In order to develop a method for testing the statistical significance of the difference in $b$-value between two earthquake groups $A$ and $B$, we must remember that the variable

$$
F=\frac{\chi_{A}^{2} / \phi_{A}}{\chi_{B}^{2} / \phi_{B}}
$$

is distributed as the $F$-distribution with $\phi_{A}$ and $\phi_{B}$ degrees of freedom. Accordingly

$$
F=\frac{b_{B} b_{0 A}}{b_{A} b_{0 B}}
$$

have the $F$-distribution with $2 s_{A}$ and $2 s_{B}$ degrees of freedom. The hypothesis $b_{0 A}=b_{0 B}$ can be tested by comparing $b_{B} / b_{A}$ (here we assume that $b_{A}<b_{B}$ ) with the $F$-value with $2 s_{A}$ and $2 s_{B}$ degrees of freedom at a given confidence level, which will be found in most textbooks on statistics.

Hitherto such statistical tests have been made according to a method of testing the difference in regression coefficient by use of a $t$-distribution (for example, MIYAMURA, 1962b). The assumptions which such a method is based on may be applicable only approxi- mately to the present problem. The new method described here requires no assumptions such as large number of data, normal distribution of the numbers of earthquakes at each magnitude class with the same variance, etc. The following is an example of the application of this method.

SUYEHIRO (1966) investigated the magnitudefrequency distributions for foreshocks and aftershocks of the great Chilean earthquake of 1960. He found an appreciable difference in $b$-value between the foreshocks and the aftershocks. The clata he used are reproduced in Table II.

Using these clata the $b$-values are redetermined from Equation (2).

Total number of foreshocks $\quad s_{F}=31$

$$
\text { " aftershocks } s_{A}=122
$$

Sum of the magnitudes of foreshocks $\sum M_{F^{\prime} i}=31 \log 3+42 \log 2$

Sum of the magnitucles of aftershocks $\sum M_{A i}=122 \log 3+78 \log 2$

Lower limit of magnitude $\quad M_{s} \fallingdotseq \log 2$ $b$ for foreshocks $\quad b_{F}=0.74$ , aftershocks $\quad b_{A}=1.18$

Since the class interval of magnitude $\Delta M$ is 
Table II. SuyenIRo's Data on the Chilean Earthquake Series of 1960

\begin{tabular}{c|c|c|c|c}
\hline $\begin{array}{c}\text { Class Interval } \\
\text { (mm in trace } \\
\text { amplitude) }\end{array}$ & $\begin{array}{c}\text { Central Value } \\
\text { of the Class }\end{array}$ & $\begin{array}{c}\text { Logarithm } \\
\text { of the } \\
\text { Central Value }\end{array}$ & $\begin{array}{c}\text { Foreshock } \\
\text { Frequency }\end{array}$ & $\begin{array}{c}\text { Aftershock } \\
\text { Frequency }\end{array}$ \\
\hline $2-4$ & 3 & $\log 3$ & 13 & 71 \\
$4-8$ & 6 & $\log 3+\log 2$ & 7 & 31 \\
$8-16$ & 12 & $\log 3+2 \log 2$ & 4 & 13 \\
$16-32$ & 24 & $\log 3+3 \log 2$ & 3 & 7 \\
$32-64$ & 48 & $\log 3+4 \log 2$ & 2 & 0 \\
$64-128$ & 96 & $\log 3+5 \log 2$ & 2 & 0 \\
\hline
\end{tabular}

$\log 2(\fallingdotseq 0.3)$, the above $b$-value should be increased by about $4 \%$. To test the significance of the difference between $b_{F}$ and $b_{A}$, we must compare

$$
b_{A} / b_{F}=1.59
$$

with the $F$-value with 62 and 244 degrees of freedom. Since

$$
F_{0.01}(62,244)=1.57 \text {, }
$$

is smaller than $b_{A} / b_{F}$, the difference between the foreshocks and aftershocks is significant when judged at the $98 \%$ confidence level. But it is no more significant at the $99 \%$ confidence level.

\section{References}

AKI, K.:

1965 Maximum Likelihood Estimate of $b$ in the Formula $\log N=a-b M$ and its Confidence Limits, Bull. Earthq. Res. Inst., 43, 237239.
MiYamura, S.:

1962a Magnitude-Frequency Relation of Earthquakes and its Bearing on Geotectonics, Proc. Japan Acad. 38, 27-30.

1962b A Note on Fennoscandian Seismicity, Geophysica, 7 , No. 4, 1-12.

SUYEHIRO, S.:

1966 Difference between Aftershocks and Foreshocks in the Relationship of Magnitude to Frequency of Occurrence for the Great Chilean Earthquake of 1960, Bull. Seism. Soc. Am. 56, 185-200.

UTSU, T.:

1965 A Method for Determining the Value of $b$ in a Formula $\log n=a-b M$ showing the Magnitude-Frequency Relation for Earthquakes, Geophys. Bull. Hokkaido Univ. 13, 99-103, (in Japanese with English summary).

1967 Some Problems of the Frequency Distribution of Earthquakes in Respect to Magnitude, Geophys. Bull. Hokkaido Univ. 17, 85-112; 18, 53-69, (in Japanese with English summary).

(Received June 10, 1967) 